\title{
Quasirelativistic theory for magnetic shielding constants. II. Gauge-including atomic orbitals and applications to molecules
}

\author{
AUTHOR(S): \\ Fukuda, R; Hada, M; Nakatsuji, H
}

CITATION:

Fukuda, R ...[et al]. Quasirelativistic theory for magnetic shielding constants. II. Gaugeincluding atomic orbitals and applications to molecules. JOURNAL OF CHEMICAL PHYSICS 2003, 118(3): 1027-1035

ISSUE DATE:

2003-01-15

URL:

http://hdl.handle.net/2433/39746

\section{RIGHT:}

Copyright 2003 American Institute of Physics. This article may be downloaded for personal use only. Any other use requires prior permission of the author and the American Institute of Physics. 


\title{
Quasirelativistic theory for magnetic shielding constants. II. Gauge-including atomic orbitals and applications to molecules
}

\author{
Ryoichi Fukuda, Masahiko Hada, and Hiroshi Nakatsujia) \\ Department of Synthetic Chemistry and Biological Chemistry, Graduate School of Engineering, \\ Kyoto University, Sakyou-ku, Kyoto 606-8501, Japan
}

(Received 10 August 2001; accepted 22 October 2002)

\begin{abstract}
Quasirelativistic theory of magnetic shielding constants based on the Douglas-Kroll-Hess transformation of the magnetic potential presented in a previous paper is extended to molecular systems that contain heavy elements. The gauge-including atomic orbital method is adapted to the quasirelativistic Hamiltonian to allow origin-independent calculations. The present theory is applied to the proton and halogen magnetic shielding constants of hydrogen halides and the ${ }^{199} \mathrm{Hg}$ magnetic shielding constants and chemical shifts of mercury dihalides and methyl mercury halides. While the relativistic correction to the magnetic interaction term has little effect on the proton magnetic shielding constants, this correction is a dominant origin of the heavy atom shifts of the magnetic shielding constants of heavy halogens and mercury. The basis set-dependence of mercury shielding constants is quite large in the relativistic calculation; it is important to use the basis functions that are optimized by the relativistic method to properly describe the relativistic effect. The relativistic correction to the magnetic interaction term is quite important for mercury dihalides in which the relativistic effects from mercury and halogen are strongly coupled. Without this correction, we obtain quite incorrect results. The origin of the ${ }^{199} \mathrm{Hg}$ chemical shifts in mercury dihalides is the spin-orbit interaction from heavy halogens. In methyl mercury halides, the paramagnetic shielding term as well as the spin-orbit interaction from heavy halogens dominates the ${ }^{199} \mathrm{Hg}$ chemical shifts. (C) 2003 American Institute of Physics. [DOI: 10.1063/1.1528934]
\end{abstract}

\section{INTRODUCTION}

Since chemistry deals with various combinations of more-than-one-hundred elements in the periodic table, the basic theory of chemistry should be able to be seamlessly applicable to all such combinations of elements. For this purpose the Schrödinger picture alone is insufficient and we have to rely on the relativistic picture based on the Dirac equation. The relativistic effect becomes more and more important as the system includes heavier and heavier elements. ${ }^{1}$

Although rigorous relativistic treatment of molecules should be based on the four-component Dirac theory, ${ }^{2}$ a formidable computational cost originating from an explicit calculation of the small component still limits its application to small molecules. Quasirelativistic (QR) theories, on the other hand, like those based on the Douglas-Kroll-Hess (DKH) transformation $^{3-6}$ and the regular approximation, ${ }^{7-10}$ can be considered as a natural extension of the nonrelativistic Schrödinger equation, and are widely accepted as computational tools in relativistic quantum chemistry.

Properties of atoms and molecules, especially magnetic properties, fairly strongly reflect the relativistic effect. $^{22-26,28-33}$ For calculating such properties, the perturbation method that takes into account the relativistic effect on the electrostatic ${ }^{11,12}$ and magnetic properties ${ }^{13-15}$ has been presented within the framework of the DKH approximation. These studies concluded that the perturbation operators must

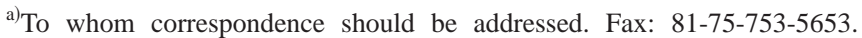
Electronic mail: hiroshi@sbchem.kyoto-u.ac.jp
}

be treated within the DKH approximation, i.e., the effect of the so-called "picture change" of the operators ${ }^{16}$ has to be taken into account for accurate calculations.

The magnetic shielding constant, in particular its electronic mechanism, is essentially the property of the resonance atom. ${ }^{17,18}$ Representing the relativistic effect with the one-electron or effective one-electron operators is a good approximation because this effect is mainly caused by the strong attraction potential of the nucleus. ${ }^{19}$ In this sense, the relativistic effect on the magnetic shielding constant of a resonance atom can be considered to originate from two sources. One is the relativistic effect from the atom that is bonded to the resonance atom, and the other is the relativistic effect from the resonance atom itself. The importance of both effects was first recognized by semiempirical works. Morishima, Endo, and Yonezawa found that the proton magnetic shielding constant of hydrogen halides was strongly affected by the spin-orbit (SO) interaction, which is one of the relativistic effects of halogens. ${ }^{20}$ Pyykkö and co-workers found out that the $\mathrm{Pb}$ magnetic shielding constant of $\mathrm{PbH}_{3}^{-}$was significantly increased by considering the relativistic effect. ${ }^{21}$

The heavy atom shift due to the SO interaction of the bonding heavy element was extensively studied in this laboratory using the $a b$ initio SO-UHF method. ${ }^{22-26}$ These studies revealed that the origin of the "normal halogen dependence" 27 lies in the SO effect of the heavy halogen. The importance of the SO interaction for chemical shifts is now well recognized through these studies. Several studies using ab initio method ${ }^{28,29}$ have been carried out to provide a 
theoretical prediction of the magnetic shielding constants of molecules that include heavy elements.

The relativistic effect from the resonance atom itself has not been studied as well. The strong relativistic effect from a heavy resonance atom requires proper treatment of the spinfree relativistic (SFR) effect, the SO interaction, and the magnetic perturbation term. ${ }^{13-15}$ Although the zeroth order regular approximation (ZORA) has been successfully used to study the magnetic shielding constants of molecules that include heavy elements, ${ }^{30,31}$ similar application of the DKH approximation is still limited. Studies using the DKH approximation were carried out by Ballard et al. ${ }^{32}$ and Fukui and Baba. ${ }^{33}$ While these studies considered the relativistic effect on the unperturbed Hamiltonian, the magnetic perturbation remained in a nonrelativistic form. Due to our recent studies, ${ }^{13,14}$ the relativistic correction on the magnetic perturbation cannot be neglected for the magnetic shielding constants of heavy elements. The so-called "picture change" effect ${ }^{11,12}$ must be considered by applying the DKH transformation to an external magnetic field. ${ }^{15}$

In this study, we apply the quasirelativistic theory of magnetic shielding constants based on the DKH method that we proposed previously ${ }^{15}$ to molecular systems. We examine the relativistic effect on the magnetic shielding constants and chemical shifts of molecules that include heavy elements. A theory that does not depend on the choice of the gauge-origin is necessary for studying the magnetic shielding constants of molecules. We use London's gauge-included atomic orbitals (GIAOs) (Refs. 34 and 35) in this study. The generalizedUHF (GUHF) wave function ${ }^{13-15}$ is used to describe the SO interaction and the spin-dependent magnetic response of the wave function. We extend the GUHF finite perturbation (FP) theory ${ }^{22,36}$ for the magnetic shielding constant to adapt the GIAO formalism, and propose the GIAO-FP-QR-GUHF method.

First, we apply the GIAO-FP-QR-GUHF method to the magnetic shielding constants of hydrogen halides. These molecules have been extensively studied with several levels of relativistic theories. $22,28,29,37,38 \mathrm{We}$ compared our results with the four-component results reported by Visscher $e t \mathrm{al}^{38}$ The dominant part of the relativistic effect on the magnetic shielding constants of proton in hydrogen halides is due to the SO interaction; ${ }^{22}$ however, both of the spin-dependent and spin-free relativistic effects and the picture change effect influence the magnetic shielding constants of heavy halogens.

Next, we address ${ }^{199} \mathrm{Hg}$ magnetic shielding constants and chemical shifts of mercury dihalides. To investigate the relativistic effect on heavy elements, it is important to use the basis functions that can adequately describe the relativistic effect. In hydrogen halides, we use uncontracted even- tempered basis functions. However, such a large set of basis functions limits the application of the theory to small systems. We propose as a relativistic basis function the contracted well-tempered Gaussian basis function ${ }^{39}$ with relativistic SCF coefficients. The well-tempered basis function has a sufficient range of exponents to describe the relativistic wave function. The exponent parameters for almost all of the elements in the periodic table are available. Moreover, their shared exponent feature is advantageous for calculating molecular properties. For the heavy atom shift of $\mathrm{Hg}$ as a heavy atom, it is important to use the basis functions optimized with relativistic theory. If we neglect the relativistic effects on the magnetic interaction and the picture change effect for $\mathrm{HgI}_{2}$ or $\mathrm{HgBr}_{2}$ in which the relativistic effects from $\mathrm{Hg}$ and halogens are coupled, the theory would lead to quite incorrect results. In mercury compounds, the origin of the ${ }^{199} \mathrm{Hg}$ chemical shifts is paramagnetic shielding and the Fermicontact terms. The present theory can adequately explain the trend of the chemical shifts of the molecules including heavy elements, and provides a good starting point for a more accurate theory that includes electron correlation effects.

\section{THEORY}

The basic quasirelativistic theory used in the present study is given in Ref. 15 (referred to as "Paper I" below), in which the DKH Hamiltonian within a magnetic field was formulated and written as

$$
H=\sum_{j}\left(E_{j}+V_{j}^{\mathrm{eff}}+H_{j}^{\mathrm{mag}}\right)+\sum_{j>k} V_{j k},
$$

where

$$
E_{j}=c\left(p_{j}^{2}+c^{2}\right)^{1 / 2}
$$

is the kinetic energy operator and $V_{j}^{\text {eff }}$ represents the effective scalar potential that includes the spin-free and SO interaction, ${ }^{5,6}$

$$
\begin{aligned}
V_{j}^{\mathrm{eff}}= & K_{j}\left(V_{j}+R_{j} \mathbf{p}_{j} V_{j} \cdot \mathbf{p}_{j} R_{j}\right) K_{j} \\
& +i \boldsymbol{\sigma}_{j} \cdot\left[K_{j} R_{j}\left(\mathbf{p}_{j} V_{j} \times \mathbf{p}_{j}\right) R_{j} K_{j}\right]-\frac{1}{2}\left[\left(W_{j}^{V}\right)^{2} E_{j}\right. \\
& \left.+2 W_{j}^{V} E_{j} W_{j}^{V}+E_{j}\left(W_{j}^{V}\right)^{2}\right] .
\end{aligned}
$$

$V_{j k}$ denotes the electron-electron interaction term that includes electron repulsion and two-electron SO interaction as

$$
V_{j k}=\frac{1}{r_{j k}}-\frac{1}{4 c^{2}}\left[\frac{\left(\mathbf{l}_{j k}+2 \mathbf{l}_{k j}\right) \cdot \boldsymbol{\sigma}_{j}}{r_{j k}^{3}}+\frac{\left(\mathbf{l}_{k j}+2 \mathbf{l}_{j k}\right) \cdot \boldsymbol{\sigma}_{k}}{r_{k j}^{3}}\right],
$$

where $\mathbf{l}_{j k}=\mathbf{r}_{j k} \times \mathbf{p}_{j}$. The magnetic interaction operator $H_{j}^{\text {mag }}$ is given by ${ }^{15}$

$$
\begin{aligned}
H_{j}^{\mathrm{mag}}= & K_{j}\left[R_{j} \boldsymbol{\sigma}_{j} \cdot \mathbf{p}_{j}\left(c \boldsymbol{\sigma}_{j} \cdot \mathbf{A}_{j}\right)+\left(c \boldsymbol{\sigma}_{j} \cdot \mathbf{A}_{j}\right) \boldsymbol{\sigma}_{j} \cdot \mathbf{p}_{j} R_{j}\right] K_{j} \\
& +\frac{1}{2}\left[\left(W_{j}^{V} W_{j}^{\mathbf{A}}-W_{j}^{\mathbf{A}} W_{j}^{V}\right) E_{j}+2\left(W_{j}^{V} E_{j} W_{j}^{\mathbf{A}}-W_{j}^{\mathbf{A}} E_{j} W_{j}^{V}\right)\right. \\
& \left.+E_{j}\left(W_{j}^{V} W_{j}^{\mathbf{A}}-W_{j}^{\mathbf{A}} W_{j}^{V}\right)\right] \\
& +\frac{1}{2}\left[\left(W_{j}^{\mathbf{A}}\right)^{2} E_{j}+2 W_{j}^{\mathbf{A}} E_{j} W_{j}^{\mathbf{A}}+E_{j}\left(W_{j}^{\mathbf{A}}\right)^{2}\right]
\end{aligned}
$$

The vector potential from the uniform magnetic field $\mathbf{B}$ and the nuclear magnetic moment $\boldsymbol{\mu}_{A}$ of nucleus $A$ is written as

$$
\mathbf{A}_{j}=\mathbf{A}_{j}^{0}+\mathbf{A}_{j}^{N},
$$

with

$$
\begin{aligned}
& \mathbf{A}_{j}^{0}=\frac{1}{2} \mathbf{B} \times \mathbf{r}_{j d}, \\
& \mathbf{A}_{j}^{N}=-\frac{1}{c^{2}} \sum_{A}^{\text {nuc }} \boldsymbol{\mu}_{A} \times \nabla_{j} G_{j A},
\end{aligned}
$$


where

$$
G_{j A}=\int \frac{w_{A}\left(R_{A}\right)}{\left|\mathbf{r}_{j}-\mathbf{R}\right|} \mathrm{d} \mathbf{R} .
$$

Here, $\mathbf{r}_{j d}=\mathbf{r}_{j}-\mathbf{d}$ is the position vector from the arbitrary gauge-origin d. $w_{A}$ represents the distribution of the nuclear magnetic moment. ${ }^{40}$ The Gaussian nucleus model $^{41-43}$ is used in this study.

The magnetic shielding tensor of nucleus $A$ in $t u$ component $(t, u=x, y, z)$ is given by ${ }^{44}$

$$
\sigma_{A, t u}=\left.\frac{\partial^{2} E}{\partial B_{t} \partial \mu_{A, u}}\right|_{\mu_{A, u}=B_{t}=0} .
$$

If we consider the change of picture effect for magnetic perturbation, the Hellmann-Feynman theorem holds for the quasirelativistic Hartree-Fock SCF wave function ${ }^{11,12}$ for the derivatives with respect to $\mu_{A},{ }_{u}$; and thus the magnetic shielding tensor can be written in the form ${ }^{22}$

$$
\begin{aligned}
\sigma_{A, t u}= & \sum_{j}^{\text {occ }}\left\{\left\langle\phi_{j}^{(0)}\left|H_{j A t u}^{(1,1)}\right| \phi_{j}^{(0)}\right\rangle\right. \\
& \left.+\frac{\partial}{\partial B_{t}}\left[\left\langle\phi_{j}^{\left(B_{t}\right)}\left|H_{j A u}^{(0,1)}\right| \phi_{j}^{\left(B_{t}\right)}\right\rangle\right]_{B_{t}=0}\right\} .
\end{aligned}
$$

The Hamiltonian is expanded in powers of $\mathbf{B}$ and $\boldsymbol{\mu}_{A}$ as

$$
\begin{aligned}
H= & H^{(0,0)}+\sum_{t} H_{t}^{(1,0)} B_{t}+\sum_{A} \sum_{u} H_{A, u}^{(0,1)} \mu_{A, u} \\
& +\sum_{A} \sum_{t u} B_{t} H_{A, t u}^{(1,1)} \mu_{A, u}+\cdots
\end{aligned}
$$

The explicit forms of $H^{(0,0)}, H^{(1,0)}, H^{(0,1)}$, and $H^{(1,1)}$ are given in Paper I. The GUHF wave function ${ }^{45,46}$ is used to describe spin-dependent properties. The GUHF orbital is written as a linear combination of atomic orbital (AO) basis functions $\chi$ as

$$
\phi_{j}=\sum_{\omega=\alpha, \beta} \sum_{\lambda} C_{\lambda j}^{\omega} \chi_{\lambda} \omega
$$

with the complex molecular orbital (MO) coefficients $C$, and spin function $\omega=\alpha$ or $\beta$. The MO coefficients and the orbital energy $\varepsilon$ are determined by solving the GUHF-SCF equations

$$
\sum_{\omega^{\prime}=\alpha, \beta} \sum_{\lambda}\left(F_{\nu \lambda}^{\omega^{\prime} \omega}-\varepsilon_{j}^{(0)} S_{\nu \lambda}^{\omega^{\prime} \omega}\right) C_{\lambda j}^{\omega(0)}=0
$$

under the orthonormality condition

$$
\sum_{\omega^{\prime} \omega=\alpha, \beta} \sum_{\nu \lambda} C_{\nu k}^{\omega^{\prime}(0) *} S_{\nu \lambda}^{\omega^{\prime} \omega} C_{\lambda j}^{\omega(0)}=\delta_{k j} .
$$

Superscript (0) represents the solutions without a magnetic field. The matrix element of the Fock operator is given by

$$
\begin{aligned}
F_{\nu \lambda}^{\omega^{\prime} \omega}= & \left\langle\chi_{\nu} \omega^{\prime}\left|h^{(0,0)}\right| \chi_{\lambda} \omega\right\rangle \\
& +\sum_{\tau \tau^{\prime}=\alpha, \beta} \sum_{\rho \sigma}\left[\left(\chi_{\nu} \omega^{\prime} \chi_{\lambda} \omega\left|V_{12}\right| \chi_{\rho} \tau^{\prime} \chi_{\sigma} \tau\right)\right. \\
& \left.-\left(\chi_{\nu} \omega^{\prime} \chi_{\sigma} \tau\left|V_{12}\right| \chi_{\rho} \tau^{\prime} \chi_{\lambda} \omega\right)\right] D_{\rho \sigma}^{\tau^{\prime} \tau},
\end{aligned}
$$

where the density matrix is given by

$$
D_{\rho \sigma}^{\tau^{\prime} \tau}=\sum_{k}^{\mathrm{occ}} C_{\rho k}^{\tau^{\prime} *} C_{\sigma k}^{\tau}
$$

The matrix elements of one-electron integrals are written as

$$
\begin{aligned}
\left\langle\chi_{\nu} \omega^{\prime}\left|h^{(0,0)}\right| \chi_{\lambda} \omega\right\rangle= & \left\langle\chi_{\nu}\left|\left(E_{p}+V^{\mathrm{SF}}\right)\right| \chi_{\lambda}\right\rangle \delta_{\omega^{\prime} \omega} \\
& +\left\langle\chi_{\nu} \omega^{\prime}\left|V^{\mathrm{SO}}\right| \chi_{\lambda} \omega\right\rangle,
\end{aligned}
$$

where $V^{\mathrm{SF}}$ and $V^{\mathrm{SO}}$ are the spin-free and SO parts of $V^{\text {eff }}$, respectively. The two-electron integral includes electron repulsion and two-electron SO interaction as

$$
\begin{aligned}
\left(\chi_{\nu} \omega^{\prime} \chi_{\lambda} \omega\left|V_{12}\right| \chi_{\rho} \tau^{\prime} \chi_{\sigma} \tau\right)= & \left(\int \mathrm{d} \mathbf{r}_{1} \mathrm{~d} \mathbf{r}_{2} \chi_{\nu}\left(\mathbf{r}_{1}\right) \chi_{\lambda}\left(\mathbf{r}_{1}\right) \frac{1}{r_{12}} \chi_{\rho}\left(\mathbf{r}_{2}\right) \chi_{\sigma}\left(\mathbf{r}_{2}\right)\right) \delta_{\omega \omega^{\prime}} \delta_{\tau \tau^{\prime}} \\
& -\frac{1}{4 c^{2}} \sum_{t=x, y, z}\left[\left(\int \mathrm{d} \mathbf{r}_{1} \mathrm{~d} \mathbf{r}_{2} \chi_{\nu}\left(\mathbf{r}_{1}\right) \chi_{\lambda}\left(\mathbf{r}_{1}\right) \frac{\left(l_{12}^{t}+2 l_{21}^{t}\right)}{r_{12}^{3}} \chi_{\rho}\left(\mathbf{r}_{2}\right) \chi_{\sigma}\left(\mathbf{r}_{2}\right)\right)\left(\omega^{\prime}\left|\sigma^{t}\right| \omega\right) \delta_{\tau \tau^{\prime}}\right. \\
& \left.+\left(\int \mathrm{d} \mathbf{r}_{1} \mathrm{~d} \mathbf{r}_{2} \chi_{\nu}\left(\mathbf{r}_{1}\right) \chi_{\lambda}\left(\mathbf{r}_{1}\right) \frac{\left(l_{21}^{t}+2 l_{12}^{t}\right)}{r_{21}^{3}} \chi_{\rho}\left(\mathbf{r}_{2}\right) \chi_{\sigma}\left(\mathbf{r}_{2}\right)\right) \delta_{\omega \omega^{\prime}}\left(\tau^{\prime}\left|\sigma^{t}\right| \tau\right)\right]
\end{aligned}
$$

The overlap integral is given by

$$
S_{\nu \lambda}^{\omega^{\prime} \omega}=\left\langle\chi_{\nu} \mid \chi_{\lambda}\right\rangle \delta_{\omega^{\prime} \omega} .
$$

To calculate magnetic shielding tensor, the response of the MOs with respect to uniform magnetic field $\mathbf{B}$ is necessary. We adapt the finite perturbation (FP) theory ${ }^{22,36}$ for the GUHF-SCF equations where a small amount of finite magnetic perturbation $B_{t}$ is applied in the SCF procedure. To allow gauge-origin-independent calculations for magnetic properties, we use GIAO functions, ${ }^{34,35}$ which are defined by

$$
\chi_{\nu}^{(\mathbf{B})}=\exp \left[-\frac{i}{2}\left(\mathbf{B} \times\left(\mathbf{R}_{\nu}-\mathbf{d}\right)\right) \cdot \mathbf{r}\right] \chi_{\nu},
$$

where $\chi_{\nu}$ is the usual real AO function that is centered at $\mathbf{R}_{\nu}$. The GUHF-MO under the magnetic field perturbation is given by the GIAO basis as 


$$
\phi_{j}^{(\mathbf{B})}=\sum_{\omega=\alpha, \beta} \sum_{\lambda} C_{\lambda j}^{\omega(\mathbf{B})} \chi_{\lambda}^{(\mathbf{B})} \omega .
$$

The coefficient $C^{(\mathbf{B})}$ is determined by solving the GIAO-FPGUHF-SCF equations

$$
\begin{aligned}
& \sum_{\omega^{\prime}=\alpha, \beta} \sum_{\lambda}\left[\left(F_{\nu \lambda}^{\omega^{\prime} \omega}-\varepsilon_{j}^{\left(B_{t}\right)} S_{\nu \lambda}^{\omega^{\prime} \omega}\right)\right. \\
& \left.\quad+B_{t}\left(H_{\nu \lambda}^{\left(B_{t}\right) \omega^{\prime} \omega}-\varepsilon_{j}^{\left(B_{t}\right)} S_{\nu \lambda}^{\left(B_{t}\right) \omega^{\prime} \omega}\right)\right] C_{\lambda j}^{\omega\left(B_{t}\right)}=0
\end{aligned}
$$

under orthonormal conditions

$$
\sum_{\omega^{\prime} \omega=\alpha, \beta} \sum_{\nu \lambda} C_{\nu k}^{\omega^{\prime}\left(B_{t}\right) *}\left(S_{\nu \lambda}^{\omega^{\prime} \omega}+B_{t} S_{\nu \lambda}^{\left(B_{t}\right) \omega^{\prime} \omega}\right) C_{\lambda j}^{\omega\left(B_{t}\right)}=\delta_{k j} .
$$

The matrix elements of the magnetic perturbation are given by

$$
\begin{aligned}
H_{\nu \lambda}^{\left(B_{t}\right) \omega^{\prime} \omega}= & \left\langle\chi_{\nu} \omega^{\prime}\left|H_{t}^{(1,0)}\right| \chi_{\lambda} \omega\right\rangle+\left\langle\chi_{\nu} \omega^{\prime}\left|f_{t, \nu \lambda}^{(1,0)} h^{(0,0)}\right| \chi_{\lambda} \omega\right\rangle \\
& +G_{t, \nu \lambda}^{(1,0) \omega^{\prime} \omega} .
\end{aligned}
$$

Since the GIAO depends on the magnetic field,

$$
\begin{aligned}
G_{t, \nu \lambda}^{(1,0) \omega^{\prime} \omega}= & \sum_{\tau \tau^{\prime}=\alpha, \beta} \sum_{\rho \sigma}\left[\left(f_{t, \nu \lambda}^{(1,0)}\left(\mathbf{r}_{1}\right) \chi_{\nu} \omega^{\prime} \chi_{\lambda} \omega\left|V_{12}\right| \chi_{\rho} \tau \chi_{\sigma} \tau^{\prime}\right)+\left(\chi_{\nu} \omega^{\prime} \chi_{\lambda} \omega\left|V_{12}\right| f_{t, \rho \sigma}^{(1,0)}\left(\mathbf{r}_{2}\right) \chi_{\rho} \tau \chi_{\sigma} \tau^{\prime}\right)\right. \\
& \left.-\left(f_{t, \nu \lambda}^{(1,0)}\left(\mathbf{r}_{1}\right) \chi_{\nu} \omega^{\prime} \chi_{\sigma} \tau^{\prime}\left|V_{12}\right| \chi_{\rho} \tau \chi_{\lambda} \omega\right)-\left(\chi_{\nu} \omega^{\prime} \chi_{\sigma} \tau^{\prime}\left|V_{12}\right| f_{t, \rho \sigma}^{(1,0)}\left(\mathbf{r}_{2}\right) \chi_{\rho} \tau \chi_{\lambda} \omega\right)\right] D_{\rho \sigma}^{\tau \tau^{\prime}}\left(B_{t}\right) .
\end{aligned}
$$

The overlap integral also includes the magnetic field as

$$
S_{\nu \lambda}^{\left(B_{t}\right) \omega^{\prime} \omega}=\left\langle f_{t, \nu \lambda}^{(1,0)} \chi_{\nu} \mid \chi_{\lambda}\right\rangle \delta_{\omega^{\prime} \omega} .
$$

The gauge-factor is defined by ${ }^{35}$

$$
f_{t, \nu \lambda}^{(1,0)}(\mathbf{r})=-\frac{i}{2}\left[\left(\mathbf{R}_{\nu}-\mathbf{R}_{\lambda}\right) \times \mathbf{r}\right]_{t} .
$$

Equations (22)-(27) summarize the GIAO-FP-QR-GUHF procedure for magnetic shielding constants. It is easier to extend a SCF program for the magnetic FP calculation at the QR-GUHF level than to extend a nonrelativistic program, because complex algebra is needed even without a magnetic field due to the presence of the SO interaction. In the DKH transformation, ${ }^{3-6}$ the evaluation of matrix elements is carried out using the matrix transformation method and by adopting the resolution of identity (RI). ${ }^{47}$ In the GIAO method, additional gauge correction terms of the DKH transformation arise in Eq. (24), due to the existence of the gauge factor in Eq. (27). The gauge factor increases the angular moment of the basis functions, ${ }^{35}$ and thus, we perform the DKH transformation with a space that includes higher angular moment set as $\{\chi+\mathbf{r} \chi\}$.

The magnetic shielding tensor can be written as

$$
\begin{aligned}
\sigma_{A, t u}= & \sum_{\omega \omega^{\prime}=\alpha, \beta} \sum_{\nu \lambda} D_{\nu \lambda}^{\omega^{\prime} \omega(0)}\left[\left\langle\chi_{\nu} \omega^{\prime}\left|H_{A t u}^{(1,1)}\right| \chi_{\lambda} \omega\right\rangle\right. \\
& \left.+\left\langle\chi_{\nu} \omega^{\prime}\left|f_{t, \nu \lambda}^{(1,0)} H_{A u}^{(0,1)}\right| \chi_{\lambda} \omega\right\rangle\right] \\
& +\sum_{\omega \omega^{\prime}=\alpha, \beta} \sum_{\nu \lambda}\left[\frac{\partial D_{\nu \lambda}^{\omega^{\prime} \omega\left(B_{t}\right)}}{\partial B_{t}}\right]_{B_{t}=0}\left\langle\chi_{\nu} \omega^{\prime}\left|H_{A, u}^{(0,1)}\right| \chi_{\lambda} \omega\right\rangle .
\end{aligned}
$$

In the FP theory, the differentiation of the density matrix is carried out numerically. With a use of the finite field of $B_{t}$ $=10^{-3}-10^{-4}$ (a.u.), the error introduced by numerical differentiation is less than the present level of accuracy com- pared to an analytical method. The first part in Eq. (28) is the diamagnetic shielding term, the second parts can be separated into the spin-free paramagnetic shielding term and the SO-induced shielding term.

\section{CALCULATIONS AND RESULTS}

As proposed in Paper I, we perform three different levels of approximations for the quasirelativistic (QR) Hamiltonians as follows:

(a) QR level 0 (QR-0): the DKH Hamiltonian with nonrelativistic magnetic interaction $H_{0}^{\mathrm{mag}}$,

$H^{\mathrm{QR}-0}=\sum_{j}\left(E_{j}+V_{j}^{\mathrm{eff}}\right)+\sum_{j>k} V_{j k}+\sum_{j} H_{0 j}^{\mathrm{mag}}$.

(b) QR level 1 (QR-1): the DKH Hamiltonian with the first-order DKH magnetic interaction $H_{1}^{\mathrm{mag}}$ and the nonrelativistic diamagnetic shielding term,

$$
\begin{aligned}
H^{\mathrm{QR}-1}= & \sum_{j}\left(E_{j}+V_{j}^{\mathrm{eff}}\right)+\sum_{j>k} V_{j k}+\sum_{j} H_{1 j}^{\mathrm{mag}} \\
& +\frac{1}{2 c^{2}} \sum_{j} h_{j}^{(1,1)} .
\end{aligned}
$$

(c) QR level 2 (QR-2): the DKH Hamiltonian with the second-order DKH magnetic interaction $H_{1}^{\mathrm{mag}}+H_{2}^{\mathrm{mag}}$,

$$
H^{\mathrm{QR}-2}=\sum_{j}\left(E_{j}+V_{j}^{\mathrm{eff}}\right)+\sum_{j>k} V_{j k}+\sum_{j}\left(H_{1 j}^{\mathrm{mag}}+H_{2 j}^{\mathrm{mag}}\right) .
$$

The GIAO-FP-QR-GUHF method is used for all levels of the QR calculations. The isotropic shielding constant, $\sigma_{N}$ $=\left(\sigma_{N, x x}+\sigma_{N, y y}+\sigma_{N, z z}\right) / 3$, is discussed. The chemical shift $\delta^{X}$ of a molecule $\mathrm{X}$ is defined by $\delta^{\mathrm{X}}=\sigma^{\text {ref }}-\sigma^{\mathrm{X}}$, where $\sigma^{\text {ref }}$ is the value of the reference molecule. The magnetic shielding constant can be decomposed into a diamagnetic shielding term, paramagnetic shielding term, Fermi contact term, and spin-dipolar term $;^{22}$ it can also be divided into the spin-free term $\left(\sigma^{\mathrm{dia}}+\sigma^{\mathrm{para}}\right)$ and the SO term, 
TABLE I. Proton and halogen magnetic shielding constants of hydrogen halides (ppm).

\begin{tabular}{|c|c|c|c|c|c|c|c|c|c|c|c|c|}
\hline & \multirow[b]{2}{*}{$\mathrm{Nuc}^{\mathrm{a}}$} & \multirow{2}{*}{$\frac{\mathrm{NR}}{\sigma^{\text {total }}}$} & \multicolumn{3}{|c|}{ QR-0 } & \multicolumn{3}{|c|}{ QR-1 } & \multicolumn{3}{|c|}{ QR-2 } & \multirow{2}{*}{$\begin{array}{r}\text { 4-comp. } \\
\sigma^{\text {total }}\end{array}$} \\
\hline & & & $\sigma^{\mathrm{dia}+\text { para }}$ & $\sigma^{\mathrm{SO}}$ & $\sigma^{\text {total }}$ & $\sigma^{\mathrm{dia}+\text { para }}$ & $\sigma^{\mathrm{SO}}$ & $\sigma^{\text {total }}$ & $\sigma^{\mathrm{dia}+\text { para }}$ & $\sigma^{\mathrm{SO}}$ & $\sigma^{\text {total }}$ & \\
\hline $\mathrm{HF}$ & $\mathrm{H}$ & 28.09 & 28.44 & 0.17 & 28.61 & 28.06 & 0.17 & 28.23 & 28.17 & 0.17 & 28.34 & 27.87 \\
\hline $\mathrm{HCl}$ & $\mathrm{H}$ & 30.54 & 30.62 & 1.02 & 31.64 & 30.56 & 1.00 & 31.56 & 30.54 & 1.01 & 31.56 & 31.00 \\
\hline $\mathrm{HBr}$ & $\mathrm{H}$ & 30.74 & 30.72 & 6.44 & 37.17 & 30.72 & 6.41 & 37.13 & 30.71 & 6.43 & 37.15 & 36.08 \\
\hline HI & $\mathrm{H}$ & 31.15 & 30.95 & 18.62 & 49.57 & 30.93 & 18.56 & 49.49 & 30.95 & 18.77 & 49.72 & 47.98 \\
\hline $\mathrm{HF}$ & $\mathrm{F}$ & 416.8 & 415.3 & 0.6 & 415.9 & 420.1 & 10.5 & 430.6 & 414.0 & 9.4 & 423.4 & 423.3 \\
\hline $\mathrm{HCl}$ & $\mathrm{Cl}$ & 957.5 & 964.7 & 4.1 & 968.8 & 968.1 & 66.9 & 1035.0 & 956.7 & 62.3 & 1018.9 & 1020.1 \\
\hline $\mathrm{HBr}$ & $\mathrm{Br}$ & 2634.9 & 2679.3 & 75.6 & 2754.9 & 2738.5 & 541.8 & 3280.3 & 2630.2 & 534.7 & 3164.9 & 3224.6 \\
\hline $\mathrm{HI}$ & I & 4540.4 & 4631.8 & 761.8 & 5393.6 & 4910.2 & 1992.4 & 6902.6 & 4525.6 & 1983.0 & 6508.5 & 6768.4 \\
\hline
\end{tabular}

${ }^{\mathrm{a}}$ Resonance nucleus.

${ }^{\mathrm{b}}$ Four-component relativistic RPA calculation (Ref. 38).

$$
\begin{aligned}
& \sigma_{A}=\sigma_{A}^{\mathrm{dia}}+\sigma_{A}^{\mathrm{para}}+\sigma_{A}^{\mathrm{SO}}(\mathrm{SD})+\sigma_{A}^{\mathrm{SO}}(\mathrm{FC}) \\
& =\sigma_{A}^{\text {dia }}+\text { para }+\sigma_{A}^{\mathrm{SO}} .
\end{aligned}
$$

\section{A. Magnetic shielding constants of hydrogen halides}

Hydrogen halides (HX) are the simplest molecular system that clearly shows the relativistic effect on the magnetic shielding constants. The chemical shift of this series of molecules is dominantly relativistic-effect origin, mainly the spin-orbit effect. ${ }^{22}$ We apply our theory first to this system as molecular applications. We use the basis sets that are the same as those used by Visscher et al. ${ }^{38}$ in the fourcomponent relativistic RPA study. The basis sets for hydrogen, fluorine, and chlorine are the uncontracted cc-pVTZ sets of Dunning and Woon. ${ }^{48,49}$ The basis sets for bromine and iodine are, respectively, the even-tempered $(23 s 16 p 12 d 3 f)$ and $(28 s 21 p 15 d 4 f)$ sets of Visscher et al. ${ }^{38}$ Experimental bond lengths, which were the same as those in Ref. 22, were used.

Calculated shielding constants and their decomposition into the spin-free term and SO term are summarized in Table I. The results with a nonrelativistic (NR) calculation and the four-component calculation by Visscher et $\mathrm{al}^{38}$ with relativistic RPA are also shown. The proton magnetic shielding constants of HX gives a typical example of the relativistic effect by bonding heavy elements X. It has been well documented that the SO interaction of the heavy halogen increases the magnetic shielding of proton. ${ }^{20,22,28,29}$ This SO effect from the bonding halogen is the origin of the ${ }^{1} \mathrm{H}$ chemical shifts of hydrogen halides. The SFR effect does not affect the proton shielding constants because the SFR effect is rather local compared to the SO interaction. ${ }^{32,38} \mathrm{By}$ comparing the QR-2 results with the QR-1 results, we can see that the relativistic correction of the magnetic interaction does not affect the shielding constants of hydrogen. According to these results, we can conclude that the SO interaction is the dominant relativistic effect for the proton magnetic shielding constants of hydrogen halides. The results with QR-2 are about 1.7 and $1.1 \mathrm{ppm}$ larger than the results with the four-component method for iodide and bromide, respectively. This discrepancy is thought to be a gauge-error: A common gauge-origin was located at the halogen atom in the four-component calculations. With this choice of origin, the derivatives of hydrogen basis functions (first-order basis functions) $)^{50}$ are necessary to reduce the origin-dependence. Tight $p$-functions that correspond to the derivatives of inner $s$-functions should be added for hydrogen to reduce the gauge-error of $\sigma^{\mathrm{SO}}$.

The halogen magnetic shielding constant of HX shows the heavy atom shielding due to the heavy atom itself. As we pointed out in Paper I, the dominant origin of the heavy atom shift of heavy atom (HAHA) (Ref. 21) effect is the relativistic correction of the magnetic interaction, which is considered in the QR-1 and QR-2 approximations. The present results for HX are consistent with the HAHA mechanism proposed in Paper I. The QR-0 approximation provides $\sigma^{\text {SO }}$ as a relativistic correction to the NR value. This correction is important for heavy halides and is $14 \%$ and $3 \%$ of the total values in iodide and bromide, respectively. However, this correction in iodide is $34 \%$ of the total relativistic correction that can be obtained by the four-component theory. Most of the relativistic correction is attributed to $\sigma^{\mathrm{SO}}$ at the $\mathrm{DKH}$ level that is considered by QR2. To reproduce the fourcomponent theory for the magnetic shielding constants of heavy elements, we should consider the SO interaction and the relativistic magnetic interaction. The QR-2 results are 98\% and $96 \%$ of the four-component results in bromide and iodide, respectively. Thus the QR-2 approximation underestimates the magnetic shielding constants of heavy halides in comparison with the four-component results. This tendency is different from the results of our previous study of noble gas atoms in which the QR-2 values for $\mathrm{Kr}$ and $\mathrm{Xe}$ were about $1.5 \%$ larger than the DHF values. ${ }^{15}$ In these cases, the gauge-error should be small. This tendency can be explained by the difference in the direction of the error of $\sigma^{\text {para }}$ and $\sigma(\mathrm{FC})$ in the $\mathrm{QR}-2$ approximation. In noble gases, $\sigma^{\text {para }}$ is always positive, while $\sigma^{\text {para }}$ is negative in halogens of hydrogen halides. $\sigma(\mathrm{FC})$ is positive in both cases. If we assume that the QR-2 overestimates the absolute value of $\sigma^{\text {para }}$ and underestimates that of $\sigma(\mathrm{FC})$, the errors from $\sigma^{\text {para }}$ and $\sigma(\mathrm{FC})$ may almost cancel in noble gases to give a small positive error. In halogens, however, errors from $\sigma^{\text {para }}$ and $\sigma(\mathrm{FC})$ accumulate to give relatively large negative errors. The magnetic shielding constant in the DHF is a single term, and decomposition into $\sigma^{\text {para }}$ and $\sigma(\mathrm{FC})$ as in a twocomponent method is difficult. It is difficult to estimate the accuracy of each decomposed term. 
TABLE II. ${ }^{199} \mathrm{Hg}$ magnetic shielding constants of mercury dihalides and their orbital decomposition analysis (ppm). ${ }^{a}$

\begin{tabular}{|c|c|c|c|c|c|c|c|c|c|c|c|}
\hline \multirow[b]{2}{*}{ Molecule } & \multirow[b]{2}{*}{ Orbital $^{b}$} & \multirow{2}{*}{$\frac{\mathrm{NR}}{\sigma^{\text {total }}}$} & \multicolumn{3}{|c|}{ QR-0 } & \multicolumn{3}{|c|}{ QR-1 } & \multicolumn{3}{|c|}{ QR-2 } \\
\hline & & & $\sigma^{\text {dia }+ \text { para }}$ & $\sigma^{\mathrm{SO}}$ & $\sigma^{\text {total }}$ & $\sigma^{\mathrm{dia}+\text { para }}$ & $\sigma^{\mathrm{SO}}$ & $\sigma^{\text {total }}$ & $\sigma^{\mathrm{dia}+\text { para }}$ & $\sigma^{\mathrm{SO}}$ & $\sigma^{\text {total }}$ \\
\hline \multicolumn{12}{|c|}{ Nonrelativistic basis set } \\
\hline \multirow{3}{*}{$\mathrm{HgCl}_{2}$} & core & 9303.2 & 10215.2 & -115.2 & 10100.0 & 10638.1 & 2180.8 & 12818.9 & 9524.1 & 2112.7 & 11636.8 \\
\hline & valence & -1777.6 & -2114.6 & 294.4 & 1820.2 & -1802.9 & 98.8 & -1709.1 & -1809.5 & 100.0 & -1709.5 \\
\hline & total & 7525.6 & 8100.6 & 179.2 & 8279.8 & 8835.2 & 2274.6 & 11109.8 & 7714.6 & 2212.7 & 9927.3 \\
\hline \multirow[t]{3}{*}{$\mathrm{HgBr}_{2}$} & core & $\overline{9319.4}$ & 10221.2 & 82.0 & $1 \overline{0303.2}$ & 10649.3 & 2256.4 & $\overline{12905.7}$ & 9530.5 & 2188.3 & 11718.8 \\
\hline & valence & -1598.4 & -1875.7 & 1448.5 & -427.2 & -1576.6 & 562.9 & -1013.6 & -1578.1 & 569.6 & -1008.5 \\
\hline & total & 7730.0 & 8345.5 & 1530.5 & 9876.0 & 9072.7 & 2819.3 & 11892.1 & 7952.4 & 2757.9 & 10710.3 \\
\hline \multirow[t]{3}{*}{$\mathrm{HgI}_{2}$} & core & $\overline{9267.4}$ & 10148.8 & 193.1 & $1 \overline{0341.9}$ & 10589.5 & 2303.2 & $\overline{12892.7}$ & 9470.5 & 2235.0 & 11705.5 \\
\hline & valence & -2033.8 & -2343.2 & 2385.4 & 42.2 & -1960.2 & 927.4 & -1032.8 & -1962.0 & 937.2 & -1024.8 \\
\hline & total & 7233.6 & 7805.6 & 2578.5 & 10384.1 & 8629.3 & 3230.6 & 11859.9 & 7508.5 & 3172.2 & 10680.7 \\
\hline \multicolumn{12}{|c|}{ Relativistic basis set } \\
\hline \multirow[t]{3}{*}{$\mathrm{HgCl}_{2}$} & core & 9159.2 & 10749.3 & 3243.2 & 13992.5 & 11465.5 & 6669.6 & 18135.1 & 9996.7 & 6429.0 & 16425.7 \\
\hline & valence & -1763.7 & -3550.2 & 2874.8 & -675.4 & -2508.2 & -195.0 & -2703 & -2523.5 & 38.9 & -2484.6 \\
\hline & total & 7395.5 & 7199.1 & 6118.0 & 13317.0 & 8957.3 & 6474.6 & 15431.9 & 7473.2 & 6467.9 & 13941.1 \\
\hline \multirow[t]{3}{*}{$\mathrm{HgBr}_{2}$} & core & $\overline{9166.9}$ & 10748.5 & 4291.5 & $\overline{15040.0}$ & 11460.8 & 6824.2 & $\overline{18285.0}$ & 10000.7 & 6569.3 & 16570.0 \\
\hline & valence & -1641.4 & -3474.9 & 23729.3 & 20254.4 & -2344.2 & 1462.2 & -884.1 & -2375.7 & 2040.0 & -335.7 \\
\hline & total & 7525.5 & 7273.6 & 28020.8 & 35294.3 & 9116.6 & 8286.4 & 17400.9 & 7625.0 & 8609.3 & 16234.3 \\
\hline \multirow[t]{3}{*}{$\mathrm{HgI}_{2}$} & core & $\overline{9101.8}$ & 10560.0 & 7863.4 & $\overline{18423.4}$ & 11364.3 & 7068.9 & $\overline{18433.2}$ & 9883.1 & 6885.7 & 16768.8 \\
\hline & valence & -2074.2 & -4387.9 & 42510.2 & 38122.3 & -2954.5 & 3738.8 & 784.3 & -2956.2 & 3738.8 & 782.6 \\
\hline & total & 7027.6 & 6172.1 & 50373.6 & 56545.6 & 8409.8 & 10807.7 & 19217.5 & 6926.9 & 10624.5 & 17551.4 \\
\hline
\end{tabular}

${ }^{a}$ Underlined value denotes the total magnetic shielding constant of the molecule in the approximation indicated.

${ }^{\mathrm{b}}$ Orbital contribution of the magnetic shielding constant; $($ total $)=($ core $)+($ valence $)$.

\section{B. ${ }^{199} \mathrm{Hg}$ chemical shifts}

We calculated the ${ }^{199} \mathrm{Hg}$ magnetic shielding constants of $\mathrm{HgX}_{2}(\mathrm{X}=\mathrm{Cl}, \mathrm{Br}$, and $\mathrm{I})$ and $\mathrm{HgXY}(\mathrm{X}, \mathrm{Y}=\mathrm{Me}, \mathrm{Cl}, \mathrm{Br}$, and I). The basis sets are Huzinaga-Dunning double-zeta set for hydrogen, ${ }^{51,52}$ and Ahlrichs' TZ set for carbon and chlorine, ${ }^{53}$ a single $d$-polarization function is added for each set. ${ }^{54} \mathrm{An}$ $s p$-diffuse function is also added for the chlorine set. ${ }^{54}$ For heavy atoms, we use nonrelativistic and relativistic basis functions. The nonrelativistic basis set for mercury is Huzinaga's triple-zeta for valence orbitals and double-zeta for the inner shel1 ${ }^{54}$ with two $p$-polarization functions, ${ }^{54}$ and the basis sets for bromine and iodine are Huzinaga's set ${ }^{54}$ in the same contraction as mercury with a single $d$-polarization function and one $s p$-diffuse function. ${ }^{54}$ The relativistic basis set is the well-tempered set of Huzinaga and Klobukowski ${ }^{39}$ in the relativistic contracted form. The contraction coefficients are determined by the QR-SCF calculations for atoms with primitive functions. We use the segment contraction scheme $^{55,56}$ to keep the inner shell double-zeta and valence quadruple-zeta. The innermost $p$ shell for iodine and the $p$ and $d$ shells for mercury are contracted in two different patterns to describe the SO splitting of $p^{1 / 2}$ and $p^{3 / 2}$, or $d^{3 / 2}$ and $d^{5 / 2}$ orbitals. The basis functions for other orbitals are contracted by the spin-averaged QR-SCF coefficients. The energy loss due to this contraction is $<500 \mathrm{~m} E_{\mathrm{h}}$ in each atom. After contraction, the innermost four s primitives of mercury are split to give more freedom for Fermi contact interaction. One $d$-function was added for iodine and bromine, and three $p$-functions for mercury generated from the well-tempered scheme are added for polarization. The final forms of the basis sets are $(29 s 24 p 19 d 13 f) /[18 s 15 p 8 d 3 f]$, $(28 s 23 p 18 d) /[12 s 10 p 6 d]$, and $(26 s 20 p 12 d) /[10 s 8 p 5 d]$ for mercury, iodine, and bromine, respectively. The geometries of molecules are due to the experimental one. ${ }^{57-59}$

The calculated magnetic shielding constants of $\mathrm{HgX}_{2}$ $(\mathrm{X}=\mathrm{Cl}, \mathrm{Br}$, and I) with the NR, QR-1, and QR-2 theories are summarized in Table II, where the total $\sigma$ is decomposed into two terms: $\sigma$ calculated by the QR theory is decomposed into the spin-free term and the SO term. Each term is further decomposed into the core and valence orbital contributions. Twenty-six electrons, including $5 d$ and $6 s$ electrons of mercury, and the outermost $s$ and $p$ electrons of halogens, are considered valence electrons. Total $\sigma$ is the sum of the core and valence contributions.

The basis function dependence of the NR calculation is about $20 \mathrm{ppm}$ and mainly arises from the core orbital contribution. The QR calculation significantly depends on the basis functions. The choice of the basis functions affects the valence orbital contribution as well as the core orbital contribution. The SO effect from the heavy halogens cannot be described using nonrelativistic basis functions. Therefore, the high-field shifts observed in bromide and iodide cannot be reproduced with the QR-1 and QR-2 approximations. It seems that the QR-0 approximation with nonrelativistic basis functions can reproduce such high-field shifts. However, the results with the QR-0 approximation strongly depend on the choice of basis functions. Using relativistic basis functions with QR-0, the chemical shifts of heavy halides greatly overshoot the experimental values. In the calculations for noble gases, we predicted that the QR-0 approximation contributes to an error in the magnetic shielding constants of heavy atoms. The QR-0 approximation can not treat the responses of orbitals to magnetic perturbations because it does not consider the picture change effect for the magnetic vector poten- 
TABLE III. ${ }^{199} \mathrm{Hg}$ magnetic shielding constants of mercury compounds and chemical shifts (ppm).

\begin{tabular}{|c|c|c|c|c|c|c|c|c|c|c|c|c|}
\hline \multirow[b]{4}{*}{ Molecule } & \multicolumn{8}{|c|}{ Magnetic shielding constant } & \multicolumn{4}{|c|}{ Chemical shift ${ }^{\mathrm{c}}$} \\
\hline & \multicolumn{3}{|c|}{$\mathrm{NR}^{\mathrm{a}}$} & \multicolumn{5}{|c|}{ QR-2 ${ }^{\mathrm{b}}$} & \multicolumn{2}{|c|}{$\delta^{\text {calc. }}$} & \multirow[b]{3}{*}{$\delta^{\operatorname{expt}}$} & \multirow[b]{3}{*}{ Solvent ${ }^{d}$} \\
\hline & \multicolumn{2}{|c|}{ Decomposition } & \multirow[b]{2}{*}{$\sigma^{\text {total }}$} & \multicolumn{4}{|c|}{ Decomposition } & \multirow[b]{2}{*}{$\sigma^{\text {total }}$} & \multirow[b]{2}{*}{$\mathrm{NR}^{\mathrm{a}}$} & \multirow[b]{2}{*}{$\mathrm{QR}-2^{\mathrm{b}}$} & & \\
\hline & $\sigma^{\mathrm{dia}}$ & $\sigma^{\text {para }}$ & & $\sigma^{\mathrm{dia}}$ & $\sigma^{\text {para }}$ & $\sigma^{\mathrm{SO}}(\mathrm{SD})$ & $\sigma^{\mathrm{SO}}(\mathrm{FC})$ & & & & & \\
\hline \multicolumn{13}{|c|}{ Isotropic value } \\
\hline $\mathrm{HgMe}_{2}$ & 9719.4 & -3052.8 & 6666.6 & 9898.3 & -3834.3 & -381.7 & 7089.9 & 12772.2 & 0.0 & 0.0 & 0 & neat \\
\hline $\mathrm{HgMeCl}$ & 9719.4 & -2491.0 & 7228.4 & 9896.1 & -2830.0 & -226.0 & 6417.3 & 13257.4 & -561.8 & -485.2 & $-814^{\mathrm{e}}$ & $\mathrm{CH}_{2} \mathrm{Cl}_{2}$ \\
\hline $\mathrm{HgMeBr}$ & 9720.1 & -2514.6 & 7205.5 & 9897.9 & -2970.1 & -238.9 & 7036.6 & 13725.5 & -538.9 & -953.3 & $-915^{\mathrm{e}}$ & $\mathrm{CH}_{2} \mathrm{Cl}_{2}$ \\
\hline HgMeI & 9720.6 & -2699.7 & 7021.0 & 9900.1 & -3281.0 & -313.3 & 7877.5 & 14183.3 & -354.4 & -1411.1 & $-1097^{\mathrm{e}}$ & $\mathrm{CH}_{2} \mathrm{Cl}_{2}$ \\
\hline $\mathrm{HgCl}_{2}$ & 9717.0 & -2191.4 & 7525.6 & 9894.1 & -2421.0 & -166.8 & 6634.6 & 13940.0 & -859.0 & -1168.7 & $-1549^{f}$ & THF \\
\hline $\mathrm{HgBr}_{2}$ & 9719.3 & -1989.3 & 7729.9 & 9899.0 & -2274.0 & -142.6 & 8751.9 & 16234.3 & -1063.3 & -3462.1 & $-2209^{f}$ & THF \\
\hline $\mathrm{HgI}_{2}$ & 9721.0 & -2487.5 & 7233.6 & 9903.7 & -2976.6 & -286.2 & 10910.7 & 17551.6 & -567.0 & -4779.4 & $-3430^{\mathrm{f}}$ & THF \\
\hline \multicolumn{9}{|c|}{ Axial component $\left(\sigma_{\|}\right)$} & \multicolumn{4}{|c|}{ Shielding anisotropy $(\Delta \sigma)$} \\
\hline $\mathrm{HgMe}_{2}$ & 9723.7 & -74.9 & 9648.8 & 9906.3 & 318.2 & -363.7 & 6140.5 & 16001.2 & 4473.3 & 4843.6 & $7325 \pm 55^{\mathrm{g}}$ & liquid-crystal \\
\hline $\mathrm{HgMeCl}$ & 9725.2 & -45.1 & 9680.1 & 9908.5 & 433.0 & -199.1 & 5953.7 & 16096.1 & 3677.5 & 4258.0 & $5535 \pm 80^{\mathrm{g}}$ & liquid-crystal \\
\hline $\mathrm{HgMeBr}$ & 9726.7 & -42.1 & 9684.6 & 9910.0 & 432.0 & -208.3 & 5971.9 & 16105.6 & 3718.6 & 3570.0 & $5455 \pm 100^{\mathrm{g}}$ & liquid-crystal \\
\hline $\mathrm{HgMeI}$ & 9729.0 & -41.4 & 9687.6 & 9911.8 & 423.6 & -230.1 & 5898.8 & 16004.1 & 4000.0 & 2731.1 & $5480 \pm 300^{\mathrm{g}}$ & liquid-crystal \\
\hline $\mathrm{HgCl}_{2}$ & 9726.2 & 0.0 & 9726.2 & 9907.7 & 521.4 & -128.1 & 5896.4 & 16197.4 & 3300.9 & 3384.7 & & \\
\hline $\mathrm{HgBr}_{2}$ & 9727.8 & 0.0 & 9727.8 & 9909.5 & 541.2 & -90.8 & 5772.6 & 16132.5 & 2996.8 & -152.7 & & \\
\hline $\mathrm{HgI}_{2}$ & 9734.3 & 0.0 & 9734.3 & 9915.1 & 545.4 & -83.1 & 4703.0 & 15080.4 & 3751.1 & -3706.8 & & \\
\hline \multicolumn{13}{|c|}{ Perpendicular component $\left(\sigma_{\perp}\right)$} \\
\hline $\mathrm{HgMe}_{2}$ & 9717.2 & -4541.7 & 5175.5 & 9894.3 & -5910.6 & -390.7 & 7564.6 & 11157.6 & & & & \\
\hline $\mathrm{HgMeCl}$ & 9716.6 & -3713.9 & 6002.6 & 9889.9 & -4461.5 & -239.4 & 6649.1 & 11838.1 & & & & \\
\hline $\mathrm{HgMeBr}$ & 9716.8 & -3750.8 & 5966.0 & 9891.9 & -4671.1 & -254.1 & 7569.0 & 12535.6 & & & & \\
\hline HgMeI & 9716.5 & -4028.8 & 5687.6 & 9894.3 & -5133.3 & -354.9 & 8866.9 & 13273.0 & & & & \\
\hline $\mathrm{HgCl}_{2}$ & 9712.4 & -3287.1 & 6425.3 & 9887.3 & -3892.2 & -186.1 & 7003.7 & 12812.7 & & & & \\
\hline $\mathrm{HgBr}_{2}$ & 9715.0 & -2984.0 & 6731.0 & 9893.7 & -3681.6 & -168.5 & 10241.6 & 16285.2 & & & & \\
\hline $\mathrm{HgI}_{2}$ & 9714.4 & -3731.2 & 5983.2 & 9898.0 & -4737.7 & -387.7 & 14014.6 & 18787.2 & & & & \\
\hline
\end{tabular}

${ }^{a}$ Nonrelativistic calculation with nonrelativistic basis set.

${ }^{\mathrm{b}}$ Quasirelativistic (QR-2 approximation) calculation with relativistic basis set.

${ }^{c}$ Reference molecule is $\mathrm{HgMe}_{2}$.

${ }^{\mathrm{d} S o l v e n t}$ used in the experimental measurements.

${ }^{\mathrm{e}}$ Reference 60.

${ }^{\mathrm{f}}$ Reference 61.

${ }^{\mathrm{g}}$ Reference 62

tial. The delta function is explicitly involved in QR-0; thus, $\sigma(\mathrm{FC})$ is strongly dependent on the basis function. The QR-0 approximation cannot be adopted in cases in which HAHA and the SO are coupled. QR-1 tends to overestimate the contribution of core orbitals. However, this error is canceled and does not seriously affect the relative chemical shifts. Based on the QR-2 calculation with relativistic basis functions, we can clearly see that the HAHA effect expected in $\mathrm{HgCl}_{2}$ is due to $\sigma^{\text {SO }}$ from the core orbitals $(6420 \mathrm{ppm})$. The origin of the chemical shift is the valence orbital contribution; the high-field shift of heavy halides originates from the SO interaction of heavy halogens. This mechanism of the chemical shift is similar to the proton chemical shift of hydrogen halides.

Table III gives the ${ }^{199} \mathrm{Hg}$ magnetic shielding constants of $\mathrm{HgXY}(\mathrm{X}, \mathrm{Y}=\mathrm{Me}, \mathrm{Cl}, \mathrm{Br}$, and $\mathrm{I})$ and their decomposition analyses. The ${ }^{199} \mathrm{Hg}$ chemical shift referenced to $\mathrm{HgMe}_{2}$ is also presented. We previously studied the magnetic shielding constant of $\mathrm{HgMe}_{2} \cdot{ }^{13}$ The present and previous calculations (calculation level 5 in Ref. 13) differ as follows: First, the basis functions are different. The present calculation uses GIAO, while in the previous calculation a common gauge- origin was located on the $\mathrm{Hg}$ atom. The previous calculation corresponds to the QR-1 approximation of this paper, but considered also some parts of the relativistic correction to the diamagnetic term. The total shielding constant of $\mathrm{HgMe}_{2}$ in the previous study is about $200 \mathrm{ppm}$ larger than the present result. This is mainly due to the QR-1 approximation. The present result is more reliable than the previous one, but as we have shown in the $\mathrm{HgX}_{2}$ results, the trend in the chemical shift is not affected.

The correlations between the calculated and experimental chemical shifts are shown in Fig. 1. The calculated chemical shifts are in rather good agreement with the experimental trend. The chemical shifts originate in the paramagnetic shielding term and the Fermi contact term. The chemical shifts originate in the paramagnetic shielding term and the Fermi contact term. Table III also gives the axial and perpendicular magnetic shielding tensor components and shielding anisotropy $\left(\Delta \sigma=\sigma_{\|}-\sigma_{\perp}\right)$. The perpendicular component is origin of the chemical shifts. The paramagnetic shielding term is closely related to the electronegativity of the substituents. ${ }^{18}$ The Fermi contact shifts arise from the SO interaction of halogens. We can see that the mechanism of 


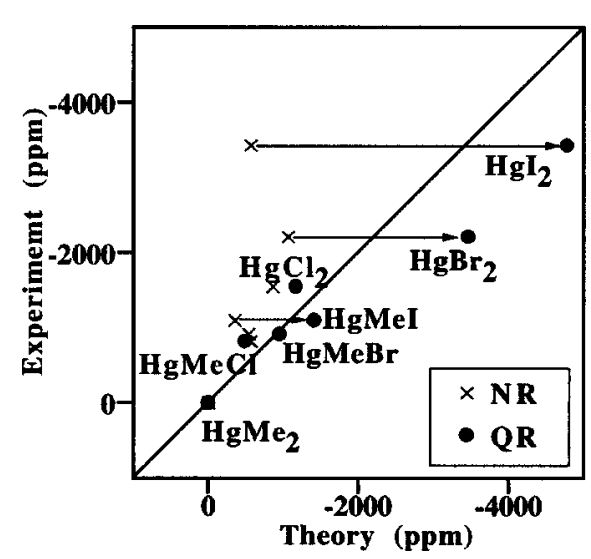

FIG. 1. Correlation between theoretical and experimental ${ }^{199} \mathrm{Hg}$ chemical shifts.

the chemical shift is similar to that for the carbon chemical shift in methyl halides. ${ }^{22,28,29}$ The calculated results do not show perfect quantitative agreement with the experimental chemical shifts. The Hartree-Fock approximation cannot adequately describe the difference between mercury-halogen and mercury-carbon chemical bonds. This discrepancy may be attributed to the electron correlation effect. The present QR-2 approximation provides a good starting point for the correlated methods. However, a consideration of the electron correlation based on the QR-0 or NR approximation is meaningless.

A series of ${ }^{199} \mathrm{Hg}$ chemical shifts was studied theoretically by Wolff et al. using ZORA and DFT. ${ }^{30}$ Since their calculation includes the electron correlation effect, their results show better agreement with experimental chemical shifts than our results. However, the absolute shielding constants with the ZORA-DFT method are smaller than those with our method. The absolute shielding constant of $\mathrm{HgMe}_{2}$ with ZORA-DFT is 8019.99 ppm, which is 4752 ppm smaller than our value. There is no difference between the $\sigma^{\text {dia }}$ and $\sigma^{\text {para }}$ values obtained by the two methods. However, the $\sigma^{\mathrm{SO}}$ with ZORA-DFT is $4250 \mathrm{ppm}$ smaller than our value. The observed chemical shift of $\mathrm{Hg}$ atom is -4638 ppm. Applying this value to the ZORA-DFT result, the obtained absolute shielding constant of $\mathrm{Hg}$ atom with ZORADFT is 12657.99 ppm. In contrast, the absolute shielding constant calculated with our QR-2 method is $16667.1 \mathrm{ppm}$. The difference in the chemical shift from the experimental result is $788.1 \mathrm{ppm}$, and the electron correlation effect should be around this value. Our QR-2 value (16667.1 ppm) is consistent with the DHF calculation for atoms. The absolute shielding constant of $\mathrm{Rn}$ with the DHF method is 19162.9 ppm and that of $\mathrm{Xe}$ is $6957.8 \mathrm{ppm} .{ }^{15}$ The cause of the difference between the present result and the ZORA-DFT result is unclear; however, the most likely reason is a difference in the basis functions. The FC term in spin-spin coupling constants with ZORA-DFT (Ref. 63) is influenced by the addition of steep basis functions. The difference between the ZORA and DKH methods is not clear.

\section{CONCLUSIONS}

In this study, we have proposed a quasirelativistic theory for the magnetic shielding constants and chemical shifts of molecular systems. This theory is based on the DKH transformation including a magnetic field that was proposed previously. We adapted the GIAO method for the finite perturbation theory and the GUHF wave function. The present GIAO-FP-QR-GUHF method was applied to the magnetic shielding constants and chemical shifts of hydrogen halides, mercury dihalides and methyl mercury halides.

For the proton magnetic shielding constants in hydrogen halides, the SO interaction is the dominant relativistic effect. The SFR term only slightly affects the magnetic shielding constants, and the picture change effect is not important. The relativistic effect on the magnetic interaction and the picture change effect are the dominant source of the heavy atom shifts of the magnetic shielding constants of the heavy halogens. The effects appear in the total shielding constants mainly through the $\sigma^{\text {SO }}$.

In the relativistic calculation, it is important to use the basis functions that are optimized by the relativistic method. Nonrelativistic basis functions cannot adequately describe the heavy atom shift induced by the SO interaction. For the shielding constants for mercury, the relativistic effect on the magnetic interaction and the picture change effect are quite important. If we neglect these effects, $\sigma^{\mathrm{SO}}$ induced by the SO interaction of heavy halogens is greatly overestimated. The HAHA effect of mercury itself comes from the SO interaction of mercury, and the heavy atom shifts of heavy halides are induced by the SO interaction of halogens. The mechanism of the ${ }^{199} \mathrm{Hg}$ chemical shift in mercury dihalides is the $\sigma^{\mathrm{SO}}$ shift induced by halogens. This mechanism is similar to that for the ${ }^{1} \mathrm{H}$ chemical shift in hydrogen halides. The mechanism of the ${ }^{199} \mathrm{Hg}$ chemical shift in methyl mercury halides originates in negative $\sigma^{\text {para }}$ and positive $\sigma^{\text {SO }}$. The dominant part of $\sigma^{\mathrm{SO}}$ is the Fermi contact term. The mechanism is similar to that for the ${ }^{13} \mathrm{C}$ chemical shifts in methyl halides.

The present method is a good approximation of the fourcomponent Dirac theory, as verified in this and previous papers, and can be applied "seamlessly" to the magnetic shielding constants of various molecules that include any of the elements in the Periodic Table. The GIAO-FP-QR-GUHF method can reproduce the NMR chemical shifts of molecules relatively qualitatively. For more accurate prediction of the chemical shifts of molecules, we must consider the electron correlation effect. The present method provides a good starting point for such studies. A relativistic many-body method for magnetic shielding constants based on the present work is currently under investigation.

\section{ACKNOWLEDGMENTS}

This work was supported by a Grant for Creative Science Research from the Ministry of Education, Science, Culture, and Sports.

${ }^{1}$ P. Pyykkö, "Relativistic Theory of Atoms and Molecules," Vols. 41 and 60, in Lecture Notes in Chemistry (Springer-Verlag, Berlin, 1986 and 1993). 
${ }^{2}$ P. A. M. Dirac, Proc. R. Soc. London, Ser. A 117, 610 (1928).

${ }^{3}$ M. Douglas and N. M. Kroll, Ann. Phys. (N.Y.) 82, 89 (1974).

${ }^{4}$ J. Sucher, Phys. Rev. A 22, 348 (1980).

${ }^{5}$ B. A. Hess, Phys. Rev. A 32, 756 (1985).

${ }^{6}$ B. A. Hess, Phys. Rev. A 33, 3742 (1986).

${ }^{7}$ E. van Lenthe, E. J. Baerends, and J. G. Snijders, J. Chem. Phys. 99, 4597 (1993).

${ }^{8}$ R. van Leeuwen, E. van Lenthe, E. J. Baerends, and J. G. Snijders, J. Chem. Phys. 101, 1272 (1994).

${ }^{9}$ E. van Lenthe, E. J. Baerends, and J. G. Snijders, J. Chem. Phys. 101, 9783 (1994).

${ }^{10}$ A. J. Sadlej, J. G. Snijders, E. van Lenthe, and E. J. Baerends, J. Chem. Phys. 102, 1758 (1995).

${ }^{11}$ V. Kellö, A. J. Sadlej, and B. A. Hess, J. Chem. Phys. 105, 1995 (1996).

${ }^{12}$ V. Kellö and A. J. Sadlej, Int. J. Quantum Chem. 68, 159 (1998).

${ }^{13}$ J. Wan, R. Fukuda, M. Hada, and H. Nakatsuji, J. Phys. Chem. A 105, 128 (2001).

${ }^{14}$ M. Hada, J. Wan, R. Fukuda, and H. Nakatsuji, J. Comput. Chem. 22, 1502 (2001).

${ }^{15}$ R. Fukuda, M. Hada, and H. Nakatsuji, J. Chem. Phys. 118, 1015 (2002), preceding paper.

${ }^{16}$ M. Barysz and A. J. Sadlej, Theor. Chim. Acta 97, 260 (1997).

${ }^{17}$ H. Nakatsuji, K. Kanda, K. Endo, and T. Yonezawa, J. Am. Chem. Soc. 106, 4653 (1984).

${ }^{18} \mathrm{H}$. Nakatsuji, Nuclear Magnetic Shielding and Molecular Structure, edited by J. A. Tossell, Vol. C-386 in NATO ASI Series (Kluwer Academic, Dordrecht, 1992).

${ }^{19}$ W. C. Ermler, R. B. Ross, and P. A. Christiansen, Adv. Quantum Chem. 19, 139 (1988)

${ }^{20}$ I. Morishima, K. Endo, and T. Yonezawa, J. Chem. Phys. 59, 3356 (1973).

${ }^{21}$ U. Edlund, T. Lejon, P. Pyykkö, T. K. Venkatachalam, and E. Buncel, J. Am. Chem. Soc. 109, 5982 (1987).

${ }^{22}$ H. Nakatsuji, H. Takashima, and M. Hada, Chem. Phys. Lett. 233, 95 (1995).

${ }^{23}$ H. Takashima, M. Hada, and H. Nakatsuji, Chem. Phys. Lett. 235, 13 (1995).

${ }^{24}$ H. Nakatsuji, T. Nakajima, M. Hada, H. Takashima, and S. Tanaka, Chem. Phys. Lett. 247, 418 (1995).

${ }^{25}$ H. Nakatsuji, M. Hada, T. Tejima, T. Nakajima, and M. Sugimoto, Chem. Phys. Lett. 249, 248 (1996).

${ }^{26}$ H. Kaneko, M. Hada, T. Nakajima, and H. Nakatsuji, Chem. Phys. Lett. 261, 1 (1996).

${ }^{27}$ R. G. Kidd, Annu. Rep. NMR Spectrosc. 10A, 1 (1980).

${ }^{28}$ V. G. Malkin, O. L. Malkina, and D. S. Salahub, Chem. Phys. Lett. 261, 355 (1996).

${ }^{29}$ J. Vaara, K. Ruud, O. Vahtras, H. Ågren, and J. Jokisaari, J. Chem. Phys. 109, 1212 (1998).

${ }^{30}$ S. K. Wolff, T. Zieglar, E. van Lenthe, and E. J. Baerends, J. Chem. Phys. 110, 7689 (1999).

${ }^{31}$ A. Rodriguez-Fortea, P. Alemany, and T. Zieglar, J. Phys. Chem. A 103, 8288 (1999).
${ }^{32}$ C. C. Ballard, M. Hada, H. Kaneko, and H. Nakatsuji, Chem. Phys. Lett. 254, 170 (1996).

${ }^{33}$ H. Fukui and T. Baba, J. Chem. Phys. 108, 3854 (1998).

${ }^{34}$ F. London, J. Phys. Radium 8, 397 (1937).

${ }^{35}$ R. Ditchfield, Mol. Phys. 27, 789 (1974).

${ }^{36}$ J. A. Pople, J. W. McIver, and N. S. Ostlund, J. Chem. Phys. 49, 2960 (1968).

${ }^{37}$ M. Hada, Y. Ishikawa, J. Nakatani, and H. Nakatsuji, Chem. Phys. Lett. 310, 342 (1999)

${ }^{38}$ L. Visscher, T. Enevoldsen, T. Saue, H. J. Aa. Jensen, and J. Oddershede, J. Comput. Chem. 20, 1262 (1999).

${ }^{39}$ S. Huzinaga and M. Klobukowski, Chem. Phys. Lett. 212, 260 (1993).

${ }^{40}$ A. Bohr and V. F. Weisskopf, Phys. Rev. 77, 94 (1950).

${ }^{41}$ O. Visser, P. J. C. Aerts, D. Hegarty, and W. C. Nieuwport, Chem. Phys. Lett. 134, 34 (1987).

${ }^{42}$ P. Chandra and B. A. Hess, Theor. Chim. Acta 88, 183 (1994).

${ }^{43}$ L. Visscher and K. G. Dyall, At. Data Nucl. Data Tables 67, 207 (1997).

${ }^{44}$ N. F. Ramsey, Phys. Rev. 78, 699 (1950).

${ }^{45}$ P. Hafner and W. H. E. Schwarz, Chem. Phys. Lett. 65, 537 (1979).

${ }^{46}$ S. K. Wolff, D. Jayatilaka, and G. S. Chandler, Chem. Phys. Lett. 103, 4562 (1995).

${ }^{47}$ B. A. Hess, R. J. Buenker, and P. Chandra, Int. J. Quantum Chem. 29, 737 (1986).

${ }^{48}$ T. H. Dunning, Jr., J. Chem. Phys. 90, 1007 (1989).

${ }^{49}$ D. E. Woon and T. H. Dunning, Jr., J. Chem. Phys. 98, 1358 (1993).

${ }^{50}$ M. Sugimoto and H. Nakatsuji, J. Chem. Phys. 102, 285 (1995).

${ }^{51}$ S. Huzinaga, J. Chem. Phys. 42, 1293 (1965).

${ }^{52}$ T. H. Dunning, Jr., J. Chem. Phys. 53, 2823 (1970).

${ }^{53}$ A. Schäfer, H. Horn, and R. Ahlrichs, J. Chem. Phys. 97, 2571 (1992).

${ }^{54}$ S. Huzinaga, J. Andzelm, M. Klobukowski, E. Radzio-Andzelm, Y. Sakai, and H. Tatewaki, Gaussian Basis Sets for Molecular Calculations (Elsevier, Amsterdam, 1984).

${ }^{55}$ S. Huzinaga, Bunshikidouhou (Iwanamisyoten, Tokyo, 1980).

${ }^{56}$ E. R. Davidson, Chem. Phys. Lett. 260, 514 (1996).

${ }^{57}$ K. Kashiwabara, S. Konaka, T. Ijima, and M. Kimura, Bull. Chem. Soc. Jpn. 46, 407 (1973).

${ }^{58}$ C. Walls, D. G. Lister, and J. Sheridan, J. Chem. Soc., Faraday Trans. 2 71, 1091 (1975)

${ }^{59}$ J. H. Callomon, E. Hirota, K. Kuchitsu, W. J. Lafferty, A. G. Maki, and C. S. Pote, Structure Data of Free Polyatomic Molecules, edited by K.-H Hellwege and A. M. Hellwege (Springer-Verlag, New York, 1976).

${ }^{60} \mathrm{R}$. K. Harris, J. D. Kennedy, and W. McFarlane, NMR and the Periodic Table, edited by R. K. Harris and B. E. Mann (Academic, New York, 1978).

${ }^{61}$ J. D. Kennedy, and W. McFarlane, Multinuclear NMR, edited by J. Mason (Plenum, New York, 1978).

${ }^{62}$ J. D. Kennedy and W. McFarlane, J. Chem. Soc., Faraday Trans. 2 72, 1653 (1976)

${ }^{63}$ J. Autschbach and T. Zieglar, J. Chem. Phys. 113, 936 (2000). 